Published in final edited form as:

Am J Ophthalmol. 2013 August ; 156(2): 228-236. doi:10.1016/j.ajo.2013.03.027.

\title{
Approach to the Diagnosis of the Uveitides
}

\author{
Douglas A. Jabs, MD, MBA ${ }^{1,2,3}$ and Jacqueline Busingye, MD ${ }^{1}$ \\ ${ }^{1}$ Department of Ophthalmology, the Mount Sinai School of Medicine \\ ${ }^{2}$ Department of Medicine, the Mount Sinai School of Medicine \\ ${ }^{3}$ Department of Epidemiology, the Johns Hopkins University Bloomberg School of Public Health
}

\begin{abstract}
Purpose-To describe an approach to diagnosing the uveitides, a collection of about 30 separate diseases characterized by intraocular inflammation.

Design-Perspective.

Methods-Integration of clinical approach with a more formal, informatics-derived approach to characterization and a Bayesian approach to laboratory testing.

Results-The patient's uveitis is characterized along several dimensions: course, laterality, anatomic location of the inflammation, morphology, presence of active infection, and the host (age, presence of a systemic disease). Posterior uveitis can be characterized further by whether it is primarily a retinitis, choroiditis, or retinal vasculitis, by whether it is paucifocal or multifocal, and by the morphology of the lesions. This characterization narrows the differential diagnosis to one or, at most, a few diseases. Laboratory screening (i.e. testing all patients) should be reserved for those diseases that can present as any type of uveitis, whereas targeted testing (i.e. testing a subset with specific features) is used selectively. Laboratory testing should be used to identify an infection (which will alter therapy) or a systemic disease that will affect the patient's health. A uveitis that is not one of the established diagnoses is designated as "undifferentiated" with the course, laterality, and anatomic location (e.g. undifferentiated bilateral chronic anterior uveitis). We avoid the term "idiopathic" uveitis as most identified non-infectious uveitic diseases are idiopathic, and most systemic diseases associated with uveitis also are idiopathic (e.g. juvenile idiopathic arthritis).
\end{abstract}

Conclusion-This approach should lead to the correct diagnosis of the specific uveitic disease in the large majority of cases without overuse of laboratory testing.

Uveitis refers to a collection of about 30 diseases characterized by intraocular inflammation (Table 1). Traditionally these diseases have been grouped by the primary anatomic location

(C) 2013 Elsevier Inc. All rights reserved.

Address: Department of Ophthalmology, the Mount Sinai School of Medicine, One Gustave L. Levy Place, Box 1183, New York, NY 10028, USA, Phone: 212-241-6752 Fax: 212-241-5764 douglas.jabs@mssm.edu.

Publisher's Disclaimer: This is a PDF file of an unedited manuscript that has been accepted for publication. As a service to our customers we are providing this early version of the manuscript. The manuscript will undergo copyediting, typesetting, and review of the resulting proof before it is published in its final citable form. Please note that during the production process errors may be discovered which could affect the content, and all legal disclaimers that apply to the journal pertain.

DISCLOSURE

Financial Disclosures: none.

Contributions to Authors in each of these areas: conception and design (DJ), analysis and interpretation (DJ), writing the article (DJ), critical revision of the article (DJ, JB), final approval of the article (DJ, JB), data collection (DJ), provision of materials (DJ), statistical expertise (DJ), obtaining funding (DJ), literature search (DJ, JB), administrative or logistic support (DJ, JB). 
of the inflammation as anterior uveitis, intermediate uveitis, posterior uveitis, and panuveitis. ${ }^{1,2}$ In the past, standardized "review of system" questionnaires often have been used to identify any symptoms of a systemic disease, and a laboratory evaluation conducted to identify the "cause" of the uveitis, an approach often termed "the etiologic diagnosis of uveitis". A refinement on this approach is the "naming-meshing" approach popularized by Smith and Nozik. ${ }^{3}$ If no underlying disease is found and a specific syndromic name cannot be given to the uveitis, it was termed "idiopathic." This approach has problems and has led to tactics such as shotgun "uveitis survey" laboratory testing (a practice deplored by uveitis experts), the idea that one should only "work up" the second attack of uveitis (because of the low yield of shotgun laboratory testing), and exhaustive searches for laboratory evidence of sarcoidosis or other systemic diseases (often using tests with a low positive predictive value) even when there is no other evident organ involvement.

Underlying these approaches is the flawed notion that uveitis typically is a manifestation of "something else" and that the "something else" must be identified regardless of cost. Also underlying this approach is the flawed concept that discovering an idiopathic systemic disease renders the uveitis not-idiopathic. For example is chronic anterior uveitis in a child without an associated systemic disease idiopathic but not idiopathic when present in a child with juvenile idiopathic arthritis? In fact, the etiology of most complex disorders is unknown (i.e. idiopathic). Risk factors can be identified and pathogenesis inferred, but except for infectious diseases, Mendelian genetic disorders, and drug- or foreign substance-related toxic or allergic reactions, most disorders are not amenable to a simple identification of one "etiology". Hence "the etiologic diagnosis of the uveitis" is a misleading concept.

A more modern approach is to recognize that the goal of the clinician is to make the diagnosis of a specific uveitic disease. The likely diagnosis can be derived from the history, examination, and for posterior uveitides, sometimes the imaging studies. Laboratory testing then is used to identify infectious diseases which cannot be identified by the morphologic picture and systemic diseases with an impact on the patient's health. Making the correct diagnosis of a specific uveitic entity is critical to management; each disease has its own course, treatment, and prognosis. The import of this approach is shown in Table 2, which lists selected posterior uveitides. Several are infectious (in this article infectious uveitides are those in which there are replicating infectious organisms) and require antimicrobial or antiviral treatment, some are spontaneously remitting diseases with a good prognosis, requiring no treatment, and several are presumed to be autoimmune (or auto-inflammatory), chronic, eye-limited disorders, requiring systemic immunosuppression. Even if one restricts the discussion of management decisions to non-infectious posterior uveitis, the management varies substantially depending on the specific disease diagnosed.

\section{Characterization of the uveitis}

The diagnosis of a uveitic entity is begun by carefully characterizing it along several dimensions (Table 3) based on the history, examination, and in selected diseases, imaging. ${ }^{4,5}$ These dimensions have been derived from the Standardization of Uveitis Nomenclature (SUN) Project, which is developing classification criteria (criteria used for research reporting) for the major uveitic diseases,${ }^{4,5}$ and adapted to clinical care. The course of the disease is determined by its onset (sudden or insidious) and duration (limited or persistent). Sudden-onset disease of limited duration is considered acute disease, whereas chronic disease typically is insidious in onset but with a persistent duration. Acute disease may be monophasic with a single, limited-in-duration episode (for research purposes defined as less than 3 months), or recurrent. The key feature of recurrent acute disease is the presence of episodes of active inflammation separated by periods of no inflammation when not on therapy. Conversely, chronic disease relapses promptly when therapy is discontinued. 
If these terms are used precisely, the often seen term "chronic/recurrent uveitis" has no meaning. ${ }^{2}$ Furthermore, precise characterization will guide therapy. Recurrent acute disease may need only treatment of acute attacks, whereas chronic disease is likely to need chronic suppressive therapy.

The second dimension is the laterality. Uveitides may be unilateral, unilateral alternating, bilateral simultaneous, or bilateral asynchronous. In unilateral alternating disease, either eye may be affected by an attack, but only one eye is affected at a time, and the attacks are episodic and recurrent in nature. Conversely, in bilateral asynchronous disease, the onset in the two eyes is not simultaneous, but both eyes remain affected after involvement begins in the second eye, and the disease typically is chronic in nature.

The third dimension is the anatomic type of uveitis: anterior, intermediate, posterior, or panuveitis. The anatomic class of uveitis is based on the primary location of the inflammatory reaction, but not on the location of any structural complications, such as macular edema. In anterior uveitis, cells are seen primarily in the anterior chamber; there may be some retrolenticular cells present (iridocyclitis), but inflammation does not extend all the way posteriorly through the vitreous. Inflammation primarily in the vitreous is termed intermediate uveitis; there may be a mild anterior chamber reaction, but there should not be anterior segment structural complications, such as posterior synechiae or peripheral anterior synechiae. If there is a substantial anterior chamber reaction with structural complications, the uveitis should be classified as both an anterior and intermediate uveitis. In posterior uveitis there are chorioretinal inflammatory lesions, and in some diseases there is an accompanying vitreous inflammatory reaction. In a panuveitis, inflammation affects the anterior chamber, vitreous, and retina/choroid but no one location predominates. ${ }^{2}$ As with any classification system, there is some arbitrariness in classification and apparent inconsistencies based on historical naming of the syndrome. Hence multifocal choroiditis with panuveitis is considered a posterior uveitis because the primary inflammation is in the choroid and the accompanying anterior chamber or vitreous reaction typically is mild.

Posterior involvement can be further subdivided based on the primary site of inflammation as a retinitis, a choroiditis, or a retinal vasculitis. Although there is some variability in the use of the term, in this context, retinal vasculitis should involve inflammation of the retinal vessels, preferably with evidence of vascular occlusion. Mere leakage should not suffice; macular edema is not retinal vasculitis, even though there is vascular leakage. ${ }^{2}$ Retinitis and choroiditis can be further described as paucifocal (a few spots) or multifocal. Sometimes the term "focal" is used interchangeably with "paucifocal" and interpreted as distinct from "multifocal". In immunologically normal hosts, retinitis typically is paucifocal and is nearly always infectious in nature (e.g. toxoplasmic retinitis, acute retinal necrosis). Multifocal retinitis does occur, but it occurs in immune compromised hosts and is infectious in nature. Choroiditis may be paucifocal or multifocal and may be either infectious or immunemediated in nature. Although presumed immune-mediated multifocal choroidopathies occur in immunologically normal hosts (e.g. birdshot chorioretinitis), infectious multifocal choroiditides typically are seen in immune compromised hosts. The choroidal lesions of a multifocal choroiditis can be further described by a short phrase that leads to the likely diagnosis. For example, the lesions of serpiginous choroiditis are amoeboid or serpentine; those of acute posterior multifocal placoid pigment epitheliopathy, placoid; those of birdshot chorioretinitis, yellow-orange ovoid; those of multifocal choroiditis with panuveitis, "punched-out" atrophic; those of punctate inner choroiditis, punctate; and those of multiple evanescent white dot syndrome, evanescent and white.

The value of this approach (which is more structured and formalized but is somewhat akin to the "naming-meshing" approach) is suggested by Table 4 . The proper characterization of the 
uveitis along these dimensions leads to a limited differential. For example, studies of anterior uveitis have shown that although only $20 \%$ of all anterior uveitis is spondyloarthropathy-associated (also known as HLA-B27-associated), nearly $80 \%$ of a patients with recurrent acute, unilateral alternating, anterior uveitis will have a spondyloarthropathy or be HLA-B27 positive. ${ }^{6}$ Of patients with HLA-B27-associated uveitis, about $60 \%$ to $75 \%$ will have an associated spondyloarthropathy, and of these, in about one-half, the spondyloarthropathy will be undiagnosed or misdiagnosed prior to the uveitis consultation. Hence the proper characterization of the uveitis can lead to the correct diagnosis of an associated systemic disease in about one-third of the patients with this uveitic disease. ${ }^{7-9}$ Other features, such as the nature of the keratic precipitates, the severity of the anterior chamber inflammation (e.g. presence of an hypopyon), the presence or absence of posterior synechiae, and the presence of other iris features (e.g. atrophy, heterochromia) assist in the diagnosis of anterior uveitis (Table 5). Fuchs heterochromic iridocyclitis (also known as Fuchs uveitis syndrome) practically is defined by the features of the "stellate" keratic precipitates, the absence of posterior synechiae, and the heterochromia. ${ }^{10}$ Although hypopyon uveitis is the classical anterior segment finding in Behçet uveitis, an hypopyon also can be seen in spondyloarthropathy-associated (HLA-B27associated) uveitis and in certain drug reactions producing uveitis (e.g. rifabutin). ${ }^{11-13}$ In fact, in the United States, because of the much greater prevalence of the spondyloarthropathies than of Behçet disease, a patient presenting with hypopyon uveitis is more likely to have spondyloarthropathy-associated uveitis. ${ }^{11}$ However, in the Middle East and the Far East, the situation is reversed due to the greater prevalence of Behçet disease. A study using anterior chamber paracentesis for polymerase chamber reaction (PCR) analysis for viral DNA demonstrated that classic appearing "herpetic uveitis" with sectoral iris atrophy nearly always (>95\%) was herpetic uveitis on PCR testing. ${ }^{14}$ Hence PCR testing will have value in uncertain cases, but in typical cases the diagnosis can be made on the clinical features.

Similarly, the posterior uveitides typically can be diagnosed based on the history and examination, sometimes abetted by imaging, particularly fluorescein angiography and sometimes indocyanine green angiography. In Table 6 , birdshot chorioretinitis and punctate inner choroiditis are contrasted based on their features along these dimensions. ${ }^{4,15}$ As one can see, the nature of the spots distinguishes between the two diseases. In the case of serpiginous choroiditis, the fluorescein angiogram (lesions block early and stain late at the borders) is very helpful in diagnosis, especially if the lesions are not adjacent to the disc, as can happen with early disease. In contrast to serpiginous choroiditis, the fluorescein angiographic appearance of the lesions of acute posterior multifocal placoid pigmentary epitheliopathy is one of early blockage and late diffuse staining of the lesions. In early birdshot chorioretinitis, the indocyanine green angiogram may show many more spots than the clinical examination making the diagnosis of a multifocal choroiditis more evident.

Table 1 lists the more commonly identified uveitic diseases and classifies them based on whether they are infectious in nature, associated with a systemic disease, or are eye-limited and presumed to be immune-mediated. It is evident that certain infections, such as syphilis and Lyme disease, can cause various uveitic patterns, and need to be excluded, as their treatment is fundamentally different (antibiotics vs. anti-inflammatory medications). Similarly, as noted above, the diagnosis of a previously undiagnosed systemic disease may have import for the patient's systemic health, even if it does not directly affect the management of the eye disease. How then should one describe a uveitis which does not fit one of the known uveitic diseases? In the past, it often would be labeled "idiopathic" uveitis, but, as noted above, that term is problematic and misleading. Our own preference is to use the term "undifferentiated" and add the descriptors related to course, laterality, and anatomic location. Hence one might diagnose an undifferentiated, bilateral, chronic anterior uveitis or 
an undifferentiated, unilateral, acute anterior uveitis. The term "undifferentiated" is borrowed from rheumatology, where a connective tissue disease that cannot be characterized as one of the known rheumatic diseases or as an overlap syndrome is diagnosed as "undifferentiated connective tissue disease," a diagnosis which has its own ICD-9 code (710.90).

\section{Use of the Laboratory}

Although the history and examination generally lead to the diagnosis, there is a need for laboratory testing. In the use of the laboratory, one should distinguish between "screening", in which all patients (or perhaps all patients in a given class of uveitis) are routinely tested, and more targeted testing, in which the laboratory tests are used in a subset of patients with specific features. Screening is appropriate for certain infections, such as syphilis and Lyme disease, as they require laboratory testing to make the diagnosis, can present as nearly any type of uveitis, and have a treatment that is markedly different: antibiotics vs. antiinflammatory medications. Hence, even though syphilitic uveitis accounts for only about $1 \%$ of uveitic cases, testing is performed on nearly every case of adolescent and adult uveitis. Because in syphilitic uveitis the specific test (e.g. fluorescent treponemal antibody [FTA], microhemagglutination, Treponema pallidum [MHA-TP], or syphilis immunoglobulin G [IgG] antibody) is positive and the non-specific test (e.g. rapid plasma regain [RPR] or venereal disease research laboratory [VDRL]) negative in about one-third of cases of ocular syphilis, screening should be performed with a specific test. ${ }^{16}$ It should be noted that the frequency of specific-test-positive, non-specific-test-negative cases in syphilitic uveitis is similar to that in late latent and tertiary syphilis. ${ }^{17}$ Lyme disease testing should be performed in endemic areas and in exposed persons, but in areas without Lyme disease, screening for it may not be necessary. Typically Lyme disease testing consists of antibody screening and Western Blot confirmation. ${ }^{18}$ Routing screening with serologic testing for other infections is of limited value due to the high prevalence of antibodies in the general population. For example, approximately $25 \%$ of the general population will have antibodies to Toxoplasma gondii, indicating previous exposure, but not disease, and over $70 \%$ will have antibodies to herpes simplex virus. ${ }^{19,20}$ Conversely, targeted testing, such as testing for Bartonella antibodies in patients with neuroretinitis, has a high yield and has value. ${ }^{21}$

Tuberculosis testing depends on the prevalence of the tuberculosis in the general population, previous tuberculosis exposure, and the disease being evaluated. A key concept in the decision to employ a test is the positive predictive value, i.e. the likelihood that a person with a positive test has the disease. Unlike sensitivity and specificity, which are characteristics of the test, the positive predictive value is a function of the test and of the disease prevalence in the population being tested. If the disease prevalence is low, screening all patients likely will result in substantial diagnostic errors. Conversely, a higher a priori probability of the disease will result in a much better performance of the test (i.e. better positive predictive value). In the United States, tuberculosis accounts for $0.2 \%$ to $0.5 \%$ of uveitis cases. The sensitivity and specificity of the PPD are $75 \%$ and $85 \%$, respectively, and of the Quantiferon-gold test are $76 \%$ and $97 \%$, respectively. ${ }^{22,23}$ If all patients with uveitis are screened for tuberculosis, the positive predictive value of a positive PPD is $1 \%$ and of a Quantiferon-gold test is $11 \% .^{22,23}$ As such, routine screening of all patients with uveitis will be misleading in the overwhelming majority of cases. There are, however, situations where tuberculosis is much more likely, and testing is appropriate. These situations include Eales' disease, a potential choroidal tuberculoma, and serpiginous-like tuberculous choroiditis. ${ }^{24}$ For example, in a patient in whom the differential diagnosis is serpiginous choroiditis vs. serpiginous-like tuberculous choroiditis (an a priori $50 \%$ chance of either disease), the positive predictive value of the of the PPD and Quantiferon-gold are $82 \%$ and $96 \%$, respectively. Furthermore, tuberculosis testing is warranted prior to immunosuppression in a 
tuberculosis-exposed patient or someone from a country with high rates of tuberculosis and in all patients prior to the use of a TNF-a inhibitor, such are infliximab or adalimumab.

It also is important to distinguish between infectious uveitides, in which there are replicating organisms, and in which antiviral or antimicrobial agents have a therapeutic role to play (e.g. syphilis), and post-infectious diseases, in which the infection has triggered a (typically) auto-inflammatory reaction. In the latter situation, the infection itself has cleared, and antimicrobial agents have little value (except for prevention of reinfection, as in the case of rheumatic fever). An example of a post-infectious disease is epidemic reactive arthritis. In this situation a gastrointestinal infection due to a limited number of infectious agents triggers an arthritic disease (often with anterior uveitis) after clearing of the gastroenteritis. The treatment is suppression of the inflammatory reaction (e.g. topical corticosteroids for the uveitis), as the uveitis is not infectious in nature. ${ }^{25}$ In this case, identification of the inciting organism will have epidemiologic and research value, but not diagnostic or therapeutic value to the individual patient.

The second use of laboratory testing in evaluating patients is to look for a systemic disease that will affect the patient's health. As noted above, patients with acute anterior uveitis, particularly recurrent acute unilateral or unilateral alternating, anterior uveitis, have a high rate of an underlying spondyloarthropathy. ${ }^{7-9}$ Because as many as one-third of these patients' spondyloarthropathy will be undiagnosed or misdiagnosed, testing these patients for HLA-B27 has value in that it may affect the management of the patient's systemic health. ${ }^{7}$ The same issues with screening and targeted testing arise in the use of the laboratory for identification of a systemic rheumatic disease. Screening all patients with uveitis with an antinuclear antibody test has a positive predictive value of $0.6 \%$ (i.e. is wrong in over $99 \%$ of cases), but does have value among patients with juvenile idiopathic arthritis, as it identifies those patients at high risk for chronic anterior uveitis. ${ }^{22}$ For other diseases, such as Behçet disease and Vogt-Koyanagi-Harada syndrome, the diagnosis is a clinical one, and there is no specific laboratory testing. ${ }^{26,27}$

Sarcoidosis deserves special mention. It accounts for 5-10\% of all cases of uveitis in large surveys. ${ }^{28-32}$ However, it can produce any type of uveitis, so it typically there is routine screening for its presence. Unfortunately, the screening for sarcoidosis often is performed with tests with a low positive predictive value and focuses on laboratory testing, rather than disease testing. The organs most often affected by sarcoidosis are the lungs ( $90 \%)$, skin ( $20 \%)$, and reticuloendothelial system (liver, spleen, and lymph nodes, collectively 25-33\%). Central nervous system sarcoidosis is potentially serious but less common $(\sim 5 \%){ }^{32}$ As such, screening should be guided by detecting organ involvement that will affect health, particularly pulmonary and hepatic organ involvement. A chest radiograph, liver enzymes, and query about skin lesions would appear to be a reasonable approach. There are some data to suggest that a chest computed tomogram may be superior to the chest radiograph for detection of pulmonary sarcoidosis, ${ }^{33}$ but the data quality are such that it remains unclear if the chest computed tomogram should replace the chest radiograph for routine screening or just be used in cases with equivocal results on chest radiograph. Although traditionally often recommended, screening with the angiotensin converting enzyme (ACE) is not warranted. The sensitivity and specificity of the ACE are $73 \%$ and $83 \%$, respectively, and those of the gallium scan are $91 \%$ and $84 \%$, respectively. ${ }^{34}$ Therefore, the positive predictive value of the ACE, when used for routine screening (as opposed to those with known sarcoidosis) is $18 \%$. Similarly the gallium scan, when used for routine screening has a positive predictive value of $22 \%$. Furthermore, the American Thoracic Society, the European Respiratory Society, and the World Association of Sarcoidosis and other Granulomatous Diseases have issued a statement that sarcoidosis is a multisystem disease characterized by granuloma formation and a predilection for pulmonary 
involvement and that the "presence of non-caseating granuloma in a single organ ... does not establish the diagnosis of sarcoidosis." 35 Therefore, a patient with chronic anterior uveitis with mutton fat keratic precipitates and a positive ACE but with no other organ involvement has an $18 \%$ chance of having sarcoidosis and should not be classified as having "limited ocular sarcoidosis". The International Workshop on Ocular Sarcoidosis addressed this problem by developing levels of certainty for ocular sarcoidosis based on systemic features and laboratory testing: definite sarcoidosis (compatible disease and biopsy confirmation), presumed sarcoidosis (uveitis and bilateral hilar adenopathy but no biopsy performed [biopsies on these patients nearly always show sarcoidosis]), and probable (uveitis, clear lungs, biopsy not done, but other compatible features and laboratory tests). Historically this last group has a $60 \%$ chance of having a positive biopsy if one is performed (and therefore of having sarcoidosis). ${ }^{36}$ Although it is useful for research and epidemiologic studies, this last category has little therapeutic import if there is no evident organ involvement other than the eye.

In general, HLA typing has limited diagnostic usefulness. As noted above, HLA-B27 typing often is useful in acute anterior uveitis, as it may have impact on the patient's systemic health. ${ }^{6,37}$ However, screening all patients with a given diagnostic class of uveitis for HLA types typically has very low positive predictive values, usually less than $20 \% .{ }^{37}$ Even for HLA-A29 and birdshot chorioretinitis, where the relative odds are over 100 of a patient with HLA-A29 to develop birdshot chorioretinitis, if all patients with posterior are screened, the positive predictive value is only $47 \% .{ }^{37}$ As such, routine screening with HLA typing has limited usefulness. However, its targeted use has value. For example, in selected situations where the a priori risk of birdshot chorioretinitis is 50\% (e.g. late-stage disease with chorioretinal scarring making morphologic diagnosis difficult), the positive predictive value goes to $92 \%$ and the negative predictive value $90 \%$, and its use is valuable. As noted above, the diagnosis of Behçet disease is a clinical one ${ }^{26}$ the positive predictive value of screening patients with posterior or panuveitis for HLA-B51 is poor, ${ }^{37}$ and its use to diagnose Behçet disease should be discouraged.

\section{Concluding comments}

In this perspective, we have addressed how to approach a patient with uveitis to identify correctly the specific disease. The discussion and tables are not exhaustive but illustrative. The approach to the uveitides recognizes that they should be approached currently as about 30 separate diseases, not as a manifestation of underlying "etiologies". The characterization of the uveitis is derived from the informatics approach used in the initial phase of the SUN Project. ${ }^{4,5}$ The approach to the diagnosis of a systemic disease is pragmatic. It is based on the concept that if there is no evident endorgan disease and there are no implications for therapy or for the patient's systemic health, then there is no reason to pursue exhaustive laboratory searches with tests of limited positive predictive value. The approach to the use of the laboratory is Bayesian, and it avoids errors of over-diagnosis. However, as every clinician knows, there are outliers - patients with atypical presentations of a disease. It is in this situation that the "art of medicine" becomes paramount -- the skillful use of clinical judgment to select additional testing in order to make the correct diagnosis. Nevertheless, the goal of this approach is to arrive at the correct diagnosis of the specific disease in the overwhelmingly majority of patients without overuse of the laboratory resulting in misleading clinical data.

\section{Acknowledgments}

Other Acknowledgements: The authors are indebted greatly to all the members of the Standardization of Uveitis Nomenclature (SUN) Working Group for the development of a standard language and standard dimensions to describe the uveitides. Members of the SUN Working Group are listed at http://research.mssm.edu/sun. 
Funding support: supported in part by grant U10-08052, "The Standardization of Uveitis Nomenclature" from the National Eye Institute, the National Institutes of Health, Bethesda, MD USA, the David M. Brown Fund for Uveitis Research, the Segura Eye Foundation, the Jane and Michael Hoffman Foundation, the Barry and Teri Volpert Foundation, and an unrestricted grant from Research to Prevent Blindness, New York, NY, USA.

\section{REFERENCES}

1. Bloch-Michel E, Nussenblatt RB. International Uveitis Study Group recommendation for the evaluation of intraocular inflammatory disease. Am J Ophthalmol. 1987; 103(2):234-235. [PubMed: 3812627]

2. Jabs DA, Nussenblatt RB, Rosenbaum JT, Standardization of Uveitis Nomenclature (SUN) Working Group. Standardization of uveitis nomenclature for reporting clinical data. Results of the First International Workshop. Am J Ophthalmol. 2005; 140(3):509-516.

3. Smith, RE.; Nozik, RA. Uveitis: A Clinical Approach to Diagnosis and Management. 3rd ed.. Lippincott Williams \& Wilkins; Michigan: 2003.

4. Okada AA, Jabs DA. The SUN project. The future is here. Arch Ophthalmol. 2012 in press.

5. Trusko BE, Thorne JE, Jabs DA, et al. The Standardization of Uveitis Nomenclature Project. Development of a clinical evidence base utilizing informatics tools and techniques. Method Inform Med. 2013:52. Epub ahead of print.

6. Rosenbaum JT. Characterization of uveitis associated with spondyloarthritis. J Rheumatol. 1989; 16(6):792-796. [PubMed: 2778762]

7. Tay-Kearney ML, Schwam BL, Lowder C, et al. Clinical features and associated systemic diseases of HLA-B27 uveitis. Am J Ophthalmol. 1996; 121(1):47-56. [PubMed: 8554080]

8. Lissen A, Meenken C. Outcomes of HLA-B27-positive and HLA-B27-negative acute anterior uveitis. Am J Ophthalmol. 1995; 120(3):351-361. [PubMed: 7661207]

9. Monnet D, Breban M, Hudry C, Dougados M, Brézin AP. Ophthalmic findings and frequency of extraocular manifestations in patients with HLA-B27 uveitis: a study of 175 cases. Ophthalmology. 2004; 111(4):802-809. [PubMed: 15051216]

10. Jones NP. Fuchs' heterochromic uveitis: an update. Surv Ophthalmol. 1993; 37(4):253-272. [PubMed: 8441952]

11. D'Alessandro LP, Forster DJ, Rao NA. Anterior uveitis and hypopyon. Am J Ophthalmol. 1991; 112(3):317-321. [PubMed: 1882942]

12. Zaidi AA, Ying GS, Daniel E, et al. Systemic Immunosuppressive Therapy for Eye Diseases Cohort Study. Hypopyon in patients with uveitis. Ophthalmology. 2010; 117(2):366-372.

13. Shafran SD, Deschênes J, Miller M, Phillips P, Toma E, MAC Study Group of the Canadian HIV Trials Network. Uveitis and pseudojaundice during a regimen of clarithromycin, rifabutin, and ethambutol. N Engl J Med. 1994; 330(6):438-439. [PubMed: 8284019]

14. Van der Lelij A, Ooijman FM, Kijlstra A, Rothova A. Anterior uveitis with sectoral iris atrophy in the absence of keratitis. A distinct clinical entity among herpetic eye diseases. Ophthalmology. 2000; 107(6):1164-1170. [PubMed: 10857838]

15. Levinson RD, Brezin A, Rothova A, Accorinti M, Holland GN. Research criteria for the diagnosis of birdshot chorioretinopathy: results of an international consensus conference. Am J Ophthalmol. 2006; 141(1):185-187. [PubMed: 16386995]

16. Tamesis RR, Foster CS. Ocular syphilis. Ophthalmology. 1990; 97(10):1281-1287. [PubMed: 2243678]

17. Harvey, AM.; Johns, RJ.; McKusick, VA.; Owens, AH.; Ross, RS. Sexually transmitted diseases. In: Harvey, Abner McGhee, editor. The Principles and Practice of Medicine. 23rd Edition. Appleton \& Lange; Michigan: 1996. p. 590-602.

18. Centers for Disease Control and Prevention. Diagnosis and treatment. Lyme Disease. Available at: http://www.cdc.gov/lyme/diagnosistreatment/ [Accessed December 7, 2012]

19. Jones JL, Kruszon-Moran D, Wilson M, et al. Toxoplasma gondii infection in the United States: seroprevalence and risk factors. Am J Epidemiol. 2001; 154(4):357-365. 15. [PubMed: 11495859] 
20. Xu F, Schillinger JA, Sternberg MR, et al. Seroprevalence and coinfection with herpes simplex virus type 1 and type 2 in the United States, 1988-1994. J Infect Dis. 2002; 185(8):1019-1024. [PubMed: 11930310]

21. Suhler EB, Lauer AK, Rosenbaum JT. Prevalence of serologic evidence of cat scratch disease in patients with neuroretinitis. Ophthalmology. 2000; 107(5):871-876. [PubMed: 10811077]

22. Rosenbaum JT, Wernick R. The utility of routine screening of patients with uveitis for systemic lupus erythematosus or tuberculosis. A Bayesian analysis. Arch Ophthalmol. 1990; 108(9):12911293. [PubMed: 2205185]

23. Albini TA, Karakousis PC, Rao NA. Interferon-gamma release assays in the diagnosis of tuberculous uveitis. Am J Ophthalmol. 2008; 146(4):486-488. [PubMed: 18804561]

24. Vasconcelos-Santos DV, Rao PK, Davies JB, Sohn EH, Rao NA. Clinical features of tuberculous serpiginouslike choroiditis in contrast to classic serpiginous choroiditis. Arch Ophthalmol. 2010; 128(7):853-858. [PubMed: 20625045]

25. Keat, A.; Toivanen, A. Reactive arthritis: clinical features and treatment. In: Hochberg, MC.; Silman, AJ.; Smolen, JS.; Weinblatt, ME.; Weisman, MH., editors. Rheumatology. 4th edition. Elsevier; Philadelphia: 2008. p. 1089-1096.

26. International Study Group for Behçet's Disease. Criteria for the diagnosis of Behçet's disease. Lancet. 1990; 335(8697):1078-1080. [PubMed: 1970380]

27. Read RW, Holland GN, Rao NR, et al. Revised diagnostic criteria for Vögt-Koyanagi-Harada disease: report of an international committee on nomenclature. Am J Ophthalmol. 2001; 131(5): 647-652. [PubMed: 11336942]

28. Jabs DA, Johns CJ. Ocular involvement in chronic sarcoidosis. Am J Ophthalmol. 1986; 102(3): 297-301. [PubMed: 3752193]

29. Henderly DE, Genstler AJ, Smith RE, Rao NA. Changing patterns of uveitis. Am J Ophthalmol. 1987; 103(2):131-136. [PubMed: 3812615]

30. Perkins ES, Folk J. Uveitis in London and Iowa. Ophthalmologica. 1984; 189(1-2):36-40. [PubMed: 6472804]

31. McCannel CA, Holland GN, Helm CJ, et al. UCLA Community-Based Uveitis Study Group. Causes of uveitis in the general practice of ophthalmology. Am J Ophthalmol. 1996; 121(1):3546. [PubMed: 8554079]

32. Jabs, D.; Nguyen, QD. Sarcoidosis. In: Ryan, SJ., editor. Retina. 4th Edition. Elsevier; St Louis: 2006. p. 1793-1802.

33. Kaiser PK, Lowder CY, Sullivan P, et al. Chest computerized tomography in the evaluation of uveitis in elderly women. Am J Ophthalmol. 2002; 133(4):499-505. [PubMed: 11931783]

34. Power WJ, Neves RA, Rodriguez A, Pedroza-Seres M, Foster CS. The value of combined serum angiotensin-converting enzyme and gallium scan in diagnosing ocular sarcoidosis. Ophthalmology. 1995; 102(12):2007-2011. [PubMed: 9098308]

35. Statement on sarcoidosis. Joint Statement of the American Thoracic Society (ATS), the European Respiratory Society (ERS) and the World Association of Sarcoidosis and Other Granulomatous Disorders (WASOG) adopted by the ATS Board of Directors and by the ERS Executive Committee, February 1999. Am J Respir Crit Care Med. 1999; 160(2):736-755. [PubMed: 10430755]

36. Herbort CP, Rao NA, Mochizuki M, members of Scientific Committee of First International Workshop on Ocular Sarcoidosis. International criteria for the diagnosis of ocular sarcoidosis: results of the first International Workshop On Ocular Sarcoidosis (IWOS). Ocul Immunol Inflamm. 2009; 17(3):160-169. [PubMed: 19585358]

37. Zamecki KJ, Jabs DA. HLA typing in uveitis: use and misuse. Am J Ophthalmol. 2010; 149(2): 189-193. [PubMed: 20103052] 


\section{Major Uveitic Diseases}

Table 1

\begin{tabular}{|c|c|c|c|}
\hline Anatomic location & Infectious & Systemic disease & No systemic disease \\
\hline \multirow[t]{4}{*}{ Anterior uveitis } & Cytomegalovirus anterior uveitis & HLA-B27-associated uveitis & Fuchs' uveitis syndrome \\
\hline & Herpes simplex anterior uveitis & $\begin{array}{l}\text { Juvenile idiopathic arthritis- } \\
\text { associated uveitis }\end{array}$ & \\
\hline & Varicella zoster anterior uveitis & Behçet disease & \\
\hline & Syphilis & Sarcoidosis & \\
\hline \multirow[t]{2}{*}{ Intermediate } & Syphilis & Multiple sclerosis-associated uveitis & Pars planitis \\
\hline & Lyme disease & Sarcoidosis & \\
\hline \multirow[t]{8}{*}{ Posterior uveitis } & Toxoplasmic retinitis & Sarcoidosis & Serpiginous choroiditis \\
\hline & Cytomegalovirus retinitis & & $\begin{array}{l}\text { Acute posterior multifocal placoid pigmen } \\
\text { epitheliopathy }\end{array}$ \\
\hline & Acute retinal necrosis & & Multiple evanescent white dot syndrome \\
\hline & Progressive outer retinal necrosis & & Birdshot chorioretinitis \\
\hline & $\begin{array}{l}\text { Diffuse unilateral subacute } \\
\text { neuroretinitis }\end{array}$ & & Multifocal choroiditis with panuveitis \\
\hline & Syphilis & & Punctate inner choroiditis \\
\hline & Lyme disease & & $\begin{array}{l}\text { Relentless placoid choroiditis } \\
\text { ("ampiginous") }\end{array}$ \\
\hline & $\begin{array}{l}\text { Tuberculosis } \\
\text { Bartonella neuroretinitis }\end{array}$ & & \\
\hline \multirow[t]{2}{*}{ Panuveitis } & Syphilis & Behçet disease & Sympathetic ophthalmia \\
\hline & Lyme disease & $\begin{array}{l}\text { Vögt-Koyanagi-Harada disease } \\
\text { Sarcoidosis }\end{array}$ & \\
\hline
\end{tabular}


Table 2

Pathogenesis, Course, and Treatment Approach of Selected Posterior Uveitides

\begin{tabular}{|c|c|c|c|}
\hline Disease & Pathogenesis & Course & Treatment \\
\hline Toxoplasmic retinitis & Parasitic infection & Recurrent acute & Antimicrobial agents \\
\hline Cytomegalovirus retinitis & Viral infection & Chronic & Antiviral agents \\
\hline Acute retinal necrosis & Viral infection & Monophasic acute & Antiviral agents \\
\hline Serpiginous-like tuberculous choroiditis & Mycobacterial infection & Chronic & Antimicrobial agents \\
\hline Acute posterior multifocal placoid pigment epitheliopathy & Unknown & Self-limited & None, good prognosis \\
\hline Multiple evanescent white dot syndrome & Unknown & Self-limited & None, good prognosis \\
\hline Serpiginous choroiditis & Presumed immune-mediated & Chronic & Immunosuppression \\
\hline Birdshot chorioretinitis & Presumed immune-mediated & Chronic & Immunosuppression \\
\hline Multifocal choroiditis with panuveitis & Presumed immune-mediated & Chronic & Immunosuppression \\
\hline Punctate inner choroiditis & Presumed immune-mediated & $\begin{array}{l}\text { Monophasic acute, } \\
\text { recurrent acute, or } \\
\text { chronic }\end{array}$ & $\begin{array}{l}\text { Variable, none to } \\
\text { immunosuppression }\end{array}$ \\
\hline
\end{tabular}


Table 3

Dimensions Characterizing Uveitis*

\begin{tabular}{|ll|}
\hline Dimension & Examples \\
\hline Course & Acute, monophasic v recurrent acute v chronic \\
Laterality & $\begin{array}{l}\text { Unilateral vs. unilateral alternating vs. bilateral, asynchronous vs. bilateral } \\
\text { simultaneous }\end{array}$ \\
Anatomic & Anterior vs. intermediate vs. posterior v panuveitis \\
Morphology & $\begin{array}{l}\text { Retinitis vs. choroiditis } \\
\text { Paucifocal vs. multifocal }\end{array}$ \\
Infection & $\begin{array}{l}\text { Toxoplasmosis vs. cytomegalovirus vs. herpes simplex virus vs. varicella zoster } \\
\text { virus vs. syphilis vs. Lyme disease vs. Bartonella }\end{array}$ \\
Host/systemic disease & $\begin{array}{l}\text { Child vs. adult } \\
\text { Immune compromised (e.g. AIDS, transplant) vs. immune competent }\end{array}$ \\
\hline
\end{tabular}

Adapted from reference 5. 
Table 6

Comparison of Features of Posterior Uveitides Birdshot Chorioretinitis and Punctate Inner Choroiditis

\begin{tabular}{|c|c|c|}
\hline Dimension/characterization & Birdshot chorioretinitis & Punctate inner choroiditis \\
\hline Onset & Insidious & Insidious or sudden \\
\hline Duration & Persistent & Limited or persistent \\
\hline Course & Chronic & Acute monophasic, recurrent acute, or chronic \\
\hline Laterality & Bilateral & $\begin{array}{l}\text { Unilateral, bilateral asynchronous, or bilateral } \\
\text { simultaneous }\end{array}$ \\
\hline Uveitis location & Posterior & Posterior \\
\hline Primary site inflammation & Choroid and retinal vasculature & Choroid \\
\hline Morphology & Multifocal choroiditis & Multifocal choroiditis \\
\hline \multirow[t]{2}{*}{ Descriptors of spots } & Ovoid, indistinct, $50-250 \mu \mathrm{m}$ & Punctate, round, $<50 \mu \mathrm{m}$ \\
\hline & Yellow-orange or cream-colored & Yellow \\
\hline Fundus location (2-dimensional) & Posterior \& mid-peripheral & Posterior \\
\hline Other features & Vitreous cells & No vitreous cells or haze \\
\hline Imaging (fluorescein angiogram) & $\begin{array}{l}\text { Undetectable to faintly hyperfluorescent spots } \\
\text { Retinal vascular leakage }\end{array}$ & $\begin{array}{l}\text { Hyperfluorescent spots } \\
\text { No retinal vascular leakage }\end{array}$ \\
\hline Imaging (indocyanine green angiogram) & Hypoperfused choroidal spots & Hypoperfused choroidal spots (variable) \\
\hline Systemic disease & None & None \\
\hline
\end{tabular}

